\title{
TWO-GENERATOR ARITHMETIC KLEINIAN GROUPS II
}

Dedicated to the memory of R. Lyndon and J. Ullman

\author{
F. W. GEHRING, C. MACLACHLAN AND G. J. MARTIN
}

\begin{abstract}
Extending earlier work, we establish the finiteness of the number of two-generator arithmetic Kleinian groups with one generator parabolic and the other either parabolic or elliptic. We also identify all the arithmetic Kleinian groups generated by two parabolic elements. Surprisingly, there are exactly 4 of these, up to conjugacy, and they are all torsion free.
\end{abstract}

\section{Introduction}

In earlier work [18], we established the finiteness of the number of two-generator arithmetic Kleinian groups generated by a pair of elliptic elements, verifying the conjectured finiteness of the number of such arithmetic generalised triangle groups. In this note we extend this result by proving the finiteness of the number of twogenerator arithmetic Kleinian groups with one generator parabolic and the other either parabolic or elliptic. Subsequently, we identify all the arithmetic Kleinian groups generated by two parabolic elements. Surprisingly, there are exactly 4 of these, up to conjugacy, and they are all torsion free. In particular, our results yield an elementary identification of all arithmetic hyperbolic 2-bridge knot and link complements, since the Wirtinger presentation of such groups consists of two parabolic elements with one relation.

There is a substantial literature on the topic of discrete groups generated by two parabolic elements, and in particular the question of when such groups are free. Numerical studies, particularly those of Riley [26], show that the space of all such groups (a one-dimensional complex space) is very complicated. It consists of a 'free' part with a highly fractal boundary and with numerous isolated points clustering to this boundary. Among these points are the (infinitely many) hyperbolic 2-bridge knot and link complements. Our knowledge of this space has been greatly contributed to by Keen and Series $[\mathbf{1 4}, \mathbf{1 5}]$ and others. On the arithmetic side of things, perhaps the most important known related result is that of Reid [25], asserting that the figure-8 knot complement is the only arithmetic knot complement.

Our approach here depends partly on the far less sophisticated results of Lyndon and Ullman [17] together with some easy number theory, and can therefore be regarded as elementary.

We begin with a few basic definitions and notations. A Kleinian group is a discrete subgroup of orientation-preserving isometries of hyperbolic 3-space $\mathbb{H}^{3}$.

Received 19 June 1997; revised 2 September 1997.

1991 Mathematics Subject Classification 30F40, $20 \mathrm{H} 10$.

Research supported in part by grants from the U. S. National Science Foundation and the N. Z. Marsden Fund.

Bull. London Math. Soc. 30 (1998) 258-266 
Throughout, we shall consider only non-elementary Kleinian groups, which are those that do not have an abelian subgroup of finite index. Equivalently, such groups are identified with (the Poincare extensions of) discrete groups of Möbius or conformal transformations of the Riemann sphere $\overline{\mathbb{C}}$. The orbit spaces of Kleinian groups are the hyperbolic 3-orbifolds or, if the Kleinian group is torsion free, hyperbolic 3 -manifolds. We use $[\mathbf{1}, \mathbf{2 0}, \mathbf{2 3}, \mathbf{3 0}]$ as basic references for the theory of discrete groups and hyperbolic spaces.

The elements of a Kleinian group, other than the identity, are loxodromic (conjugate to $z \mapsto \lambda z,|\lambda| \neq 1$ ), elliptic (conjugate to $z \mapsto \lambda z,|\lambda|=1$ ) or parabolic (conjugate to $z \mapsto z+1$ ).

We associate with each Möbius transformation

$$
f=\frac{a z+b}{c z+d}, \quad a d-b c=1,
$$

a matrix

$$
A=\left(\begin{array}{ll}
a & b \\
c & d
\end{array}\right) \in \operatorname{SL}(2, \mathbb{C}),
$$

and set $\operatorname{tr}(f)$ to be the trace of the matrix $A$, noting that it is defined only up to multiplication by -1 . Let $G=\langle f, g\rangle$ be a two-generator subgroup of $\operatorname{PSL}(2, \mathbb{C})$. We associate to each such group three complex numbers called the parameters of $G$ :

$$
\operatorname{par}(\langle f, g\rangle)=(\gamma(f, g), \beta(f), \beta(g)),
$$

where

$$
\beta(f)=\operatorname{tr}^{2}(f)-4, \quad \beta(g)=\operatorname{tr}^{2}(g)-4, \quad \gamma(f, g)=\operatorname{tr}([f, g])-2,
$$

and $[f, g]=f g f^{-1} g^{-1}$ is the multiplicative commutator. These parameters are independent of the choice of matrix representatives, and determine the group $\langle f, g\rangle$ uniquely up to conjugacy whenever $\gamma(f, g) \neq 0$; note that $\gamma(f, g)=0$ implies that $f$ and $g$ have a common fixed point.

If $f$ is parabolic, then $\beta(f)=0$, while if $g$ is elliptic, then we can replace it by a suitable power which is primitive, and so we can assume that $\beta(g)=-4 \sin ^{2}(\pi / n)$, where $n$ is the order of $g$. Thus if $G=\langle f, g\rangle$ is a Kleinian group generated by two parabolics or an elliptic of order $n$ and a parabolic, then we have

$$
\operatorname{par}(G)=\left(\gamma, 0,-4 \sin ^{2}(\pi / n)\right),
$$

where we allow the possibility $n=\infty$ if $g$ is parabolic. Thus, up to conjugacy, the space of all such discrete groups is determined uniquely by the one complex parameter $\gamma(f, g)$.

Note that if $n=2$, then the subgroup $\langle f, g f g\rangle$ is generated by a pair of parabolics and has parameters $\left(\gamma(f, g)^{2}, 0,0\right)$. Conversely, if the non-elementary group $G=\left\langle f, f^{\prime}\right\rangle$ is generated by a pair of parabolics, then $G$ is of index 2 in a group $H=\langle f, h\rangle$, where $h$ has order 2 and $\gamma(f, h)^{2}=\gamma\left(f, f^{\prime}\right)$. For the above results, see [9].

\section{Arithmetic Kleinian groups}

In this section we recall some further notation and basic results from [7]. Let $G$ be a finitely generated subgroup of $\operatorname{PSL}(2, \mathbb{C})$. The trace field of $G$ is the field generated over $\mathbb{Q}$ by the set $\operatorname{tr}(G)=\{ \pm \operatorname{tr}(g): g \in G\}$. Since $G$ is finitely generated, 
the subgroup $G^{(2)}=\left\langle g^{2}: g \in G\right\rangle$ is a normal subgroup of finite index with quotient group a finite abelian 2-group. Following [21], we call

$$
k G=\mathbb{Q}\left(\operatorname{tr}\left(G^{(2)}\right)\right)
$$

the invariant trace-field of $G$. For any finite-index subgroup $G_{1}$ of a non-elementary group $G$, one can show that $\mathbb{Q}\left(\operatorname{tr}\left(G^{(2)}\right)\right) \subset \mathbb{Q}\left(\operatorname{tr}\left(G_{1}\right)\right)$; in [24] it is shown that $k G$ is an invariant of the commensurability class. Furthermore, if we define

$$
A G=\left\{\sum a_{i} \gamma_{i} \mid a_{i} \in k G, \gamma_{i} \in G^{(2)}\right\},
$$

then $A G$ is a quaternion algebra over $k G$ and is also an invariant of the commensurability class of $G[\mathbf{2 1}]$.

We next recall some facts about quaternion algebras; see [31] for details. Let $k$ be a number field, let $v$ be a place of $k$, that is, an equivalence class of valuations on $k$, and denote by $k_{v}$ the completion of $k$ at $v$. If $B$ is a quaternion algebra over $k$, then we say that $B$ is ramified at $v$ if $B \otimes_{k} k_{v}$ is a division algebra of quaternions. Otherwise, $v$ is unramified.

In case $v$ is a place associated to a real embedding of $k, B$ is ramified if and only if $B \otimes_{k} k_{v} \cong \mathscr{H}$, where $\mathscr{H}$ is the Hamiltonian division algebra of quaternions.

We now give the definition of an arithmetic Kleinian group. Let $k$ be a number field with one complex place, and let $A$ be a quaternion algebra over $k$ ramified at all real places. Next let $\rho$ be an embedding of $A$ into $M(2, \mathbb{C})$, let $\mathcal{O}$ be an order of $A$, and let $\mathcal{O}^{1}$ be the elements of norm 1 in $\mathcal{O}$. Then $\rho\left(\mathcal{O}^{1}\right)$ is a discrete subgroup of $\operatorname{SL}(2, \mathbb{C})$, and its projection to $\operatorname{PSL}(2, \mathbb{C}), P \rho\left(\mathcal{O}^{1}\right)$, is an arithmetic Kleinian group. The commensurability classes of arithmetic Kleinian groups are obtained by considering all such $P \rho\left(\mathcal{O}^{1}\right)$. Indeed, if $G$ is an arithmetic Kleinian group, then $G^{(2)}$ is a subgroup of some such $P \rho\left(\mathcal{O}^{1}\right)$. See [2] for further details.

For an arithmetic Kleinian group $G$, the arithmetic structure is recovered from $G$, as the defining field is the invariant trace field $k G$, and the defining quaternion algebra is the invariant quaternion algebra $A G$. Thus two arithmetic Kleinian groups are commensurable if and only if the quaternion algebras are isomorphic (see [19]). Thus $k G, A G$ can be used to characterise arithmetic Kleinian groups, and this can be refined for subgroups of arithmetic Kleinian groups to obtain the following (see [7]).

THEOREM 2.1. Let $G$ be a finitely generated non-elementary Kleinian subgroup of the group PSL $(2, \mathbb{C})$. Then $G$ is a subgroup of an arithmetic Kleinian group if and only if

(i) $k G$ has exactly one complex place or is totally real,

(ii) $\operatorname{tr}(G)$ consists of algebraic integers,

(iii) $A G$ is ramified at all Archimedean places other than the place corresponding to the identity embedding of $k G$.

Following [18], we define a Kleinian group $G$ to be nearly arithmetic if $G$ is a non-elementary Kleinian subgroup of an arithmetic Kleinian group and $G$ does not split as a non-trivial free product. Of course, an arithmetic Kleinian group is nearly arithmetic.

If the nearly arithmetic group $G$ contains a parabolic element $p$, then $A G$ cannot be a division algebra, as $p^{2}-I$ is nilpotent. But then, by Wedderburn's Structure Theorem, $A G$ must be isomorphic to the matrix algebra $M_{2}(k G)$. But $M_{2}(k G)$ is not 
ramified at any places, so $k G$ must be $\mathbb{Q}$ or $\mathbb{Q}(\sqrt{-d})$. In the case where $k G=\mathbb{Q}, G$ will be commensurable with a subgroup of the classical modular group and cannot then be an arithmetic Kleinian group as it will not have finite covolume. Recall that if $O_{d}$ is the ring of integers in the imaginary quadratic field $\mathbb{Q}(\sqrt{-d})$, then the arithmetic Kleinian groups $G_{d}=\operatorname{PSL}\left(2, O_{d}\right)$ are known as the Bianchi groups. Thus from the remarks above we have the following.

THeOREM 2.2. If $G$ is an arithmetic Kleinian group which contains a parabolic element, then $G$ is commensurable with a Bianchi group $G_{d}$. In particular, the invariant trace field is a complex quadratic extension of $\mathbb{Q}$.

\section{Two-generator groups}

Next we specialise to the case where $G$ is a two-generator group and, in particular, where one generator is parabolic and the other is elliptic. In these cases, both the invariant field and the invariant quaternion algebra will be readily described in terms of the parameters of the group.

It is shown in [24] that the field $k G$ coincides with the field

$$
\mathbb{Q}\left(\left\{\operatorname{tr}^{2}(g): g \in G\right\}\right)=\mathbb{Q}(\{\beta(g): g \in G\})
$$

(see also [12]). For two-generator groups, this has the following consequence [7].

Lemma 3.1. Let $G=\langle f, g\rangle$ be a Kleinian group with $\beta(f) \neq-4$ and $\beta(g) \neq-4$. Then

$$
k G=\mathbb{Q}\left(\beta(f), \beta(g), \beta\left(f g^{-1}\right)-\gamma(f, g)\right) .
$$

Corollary 3.2. Let $G=\langle f, g\rangle$ be a Kleinian group with $\beta(f) \neq-4$ and $\gamma(f, g)$ $\neq 0, \beta(f)$. If $G_{1}=\left\langle f, g f g^{-1}\right\rangle$, then

$$
k G_{1}=\mathbb{Q}(\beta(f), \gamma(f, g)) .
$$

Proof. Apply Lemma 3.1 with the generators $f$ and $g f g^{-1}$ to find

$$
k G_{1}=\mathbb{Q}\left(\beta(f), \beta\left(f g f^{-1} g^{-1}\right)-\gamma\left(f, g f g^{-1}\right)\right) .
$$

Next

$$
\beta\left(f g f^{-1} g^{-1}\right)-\gamma\left(f, g f g^{-1}\right)=\gamma(f, g)(\beta(f)+4),
$$

and the hypotheses on $\beta(f)$ and $\gamma(f, g)$ imply that $f$ and $g f g^{-1}$ have no common fixed point. Thus $k G_{1}=\mathbb{Q}(\gamma(f, g), \beta(f))$.

COROLlary 3.3. Let $G=\langle f, g\rangle$ be a Kleinian group with $\beta(f) \neq-4, \beta(g)=-4$ and $\gamma(f, g) \neq 0, \beta(f)$. Then

$$
k G=\mathbb{Q}(\gamma(f, g), \beta(f)) .
$$

Proof. As $g$ has order two, $G_{1}=\left\langle f, g f g^{-1}\right\rangle$ has index two in $\langle f, g\rangle$. Thus $k G_{1}=k G$, being an invariant of the commensurability class.

Theorem 3.4. Let $G=\langle f, g\rangle$ be a nearly arithmetic Kleinian group with $f$ parabolic and $g$ either parabolic or elliptic of order $n$. Then we have the following. 
- $n=2,3,4,6$.

- $\gamma=\gamma(f, g)$ is an algebraic integer.

- If $\gamma$ is complex, then $k G=\mathbb{Q}(\gamma)=\mathbb{Q}(\sqrt{-d})$.

- $k G$ is real if and only if $n=2$ and $\gamma \in \mathbb{Z}$. In this case, $k G=\mathbb{Q}$ and $G$ contains a Fuchsian subgroup of index 2 which is a free product of cyclics.

- If $n \neq 2$ and $\gamma$ is real, then $\gamma$ is a negative integer and $k G=\mathbb{Q}\left(\sqrt{t_{n} \gamma}\right)$, where $t_{n}=1,2,3,4$ for $n=3,4,6, \infty$ (that is, $g$ is parabolic), respectively.

Proof. Let $G \subset \Delta$, where $\Delta$ is an arithmetic Kleinian group. Since $\Delta$ contains parabolic elements, $k \Delta=\mathbb{Q}(\sqrt{-d})$, by Theorem 2.2 , so $k G=\mathbb{Q}$ or $\mathbb{Q}(\sqrt{-d})$. Since $\operatorname{tr}^{2} g \in k G, 2 \cos 2 \pi / n \in k G$. Thus $n=2,3,4,6$.

Note that $\mathbb{Q}(\gamma) \subset k G$ and that $\gamma$ is an algebraic integer by Theorem 2.1. If $\gamma$ is complex, then $\mathbb{Q}(\gamma)=k G=\mathbb{Q}(\sqrt{-d})$.

If $k G$ is real, then $G^{(2)}$ is Fuchsian. But any non-elementary Fuchsian subgroup of an arithmetic Kleinian group is a subgroup of an arithmetic Fuchsian group [19]. In addition, any non-compact Fuchsian group of finite covolume is a free product of cyclics, and the same is true of any finitely generated subgroup of such a group. Since $G^{(2)}$ is normal in $G, G$ will leave a circle or straight line in $\mathbb{C}$ invariant. Thus either $G$ is a Fuchsian group itself, or $G$ contains a Fuchsian subgroup $H$ of index 2. The first case cannot arise, as then $G$ would be a free product of cyclics. In the second case, the parabolic element $f$ will lie in $H$ since $f^{2}$ does, and since the element $g$ cannot lie in $H$, but preserves the $H$-invariant hyperbolic plane, $g$ must have order 2. If $n=2$, then $k G=\mathbb{Q}(\gamma)$. Thus if $k G$ is real, $\gamma \in \mathbb{Z}$, and conversely.

If $n \neq 2$, then $k G=\mathbb{Q}(\lambda)$, where $\lambda=\beta\left(f g^{-1}\right)-\gamma(f, g)$, by Lemma 3.1. From the trace identity

$$
\gamma=\operatorname{tr}^{2} f+\operatorname{tr}^{2} g+\operatorname{tr}^{2} f g^{-1}-\operatorname{tr}(f) \operatorname{tr}(g) \operatorname{tr}\left(f g^{-1}\right)-4,
$$

it can be shown that $\lambda$ satisfies a quadratic equation over $\mathbb{Q}(\gamma)$ with discriminant $16 t_{n} \gamma$, with $t_{n}$ as given in the statement. See, for example, [18]. Thus if $\gamma$ is real, then $k G$ is the non-real field $\mathbb{Q}\left(\sqrt{t_{n} \gamma}\right)$.

\section{Finiteness}

The finiteness up to conjugacy of the number of 2-generator arithmetic Kleinian groups we are discussing is now an easy consequence of the following elementary discreteness result of [6].

TheOREM 4.1. Let $G$ be a Kleinian group with

$$
\operatorname{par}(G)=\left(\gamma, 0,-4 \sin ^{2}(\pi / n)\right) .
$$

If $\gamma$ lies outside the disk

$$
|z|<4(1+\cos (\pi / n))^{2},
$$

then $G$ is discrete and isomorphic to the free product of cyclics $\langle f\rangle *\langle g\rangle$.

THEOREM 4.2. Up to conjugacy, there are only finitely many nearly arithmetic Kleinian groups (and hence finitely many arithmetic Kleinian groups) generated by a parabolic and an elliptic element or by a pair of parabolic elements. 
Proof. Let $G=\langle f, g\rangle$ be such a group. We may assume that $g$ is a primitive elliptic. Then $\operatorname{par}(G)=\left(\gamma, 0,-4 \sin ^{2}(\pi / n)\right), n \leqslant 6$ and $\gamma$ is a rational or quadratic integer. Moreover, since $G$ does not split as the free product of cyclics, $\gamma$ lies within the disk $|z|<4(1+\cos (\pi / 6))^{2}<16$. There are only finitely many such $\gamma$, and therefore up to conjugacy at most finitely many such groups.

We note that one can obtain a bound on the number of such groups, since it is easy to enumerate the possibilities for $\gamma$. However, we believe that there are rather fewer groups than these estimates might suggest. The identification of exactly which possibilities occur awaits a rather more precise description of the free part of the appropriate spaces, and methods for identifying infinite-index subgroups of Bianchi groups. We return to this in a subsequent note [5].

\section{Freeness and arithmeticity}

We now determine all nearly arithmetic groups with two parabolic generators. Recall that any Kleinian group with two parabolic generators is a subgroup of index 2 in a group $\langle f, g\rangle$ where $f$ is parabolic and $g$ has order 2 .

We make use of a nice result of Lyndon and Ullman [17] ((i) and (ii) in the theorem below), extended by a result of Ignatov [13] ((iii) in the theorem).

THEOREM 5.1. Let $f$ be parabolic and let $g$ be elliptic of order 2 .

(i) Let $K_{1}$ be the convex hull of the set consisting of the disk centre the origin of radius 2 together with the two points \pm 4 . If $\gamma(f, g) \notin K_{1}$, then $\langle f, g\rangle$ is discrete and isomorphic to the free product $\mathbb{Z} * \mathbb{Z}_{2}$.

(ii) Let $K_{2}$ be the four disks $|z \pm i|<1$ and $|z \pm 2|<2$. If $\gamma(f, g) \notin K_{2}$, then $\langle f, g\rangle$ is discrete and isomorphic to the free product $\mathbb{Z} * \mathbb{Z}_{2}$.

(iii) Let $K_{3}$ be the disk $|z| \leqslant 2$ together with the region $|\mathfrak{I}(z)|<1$. If $\gamma(f, g) \notin K_{3}$, then $\langle f, g\rangle$ is discrete and isomorphic to the free product $\mathbb{Z} * \mathbb{Z}_{2}$.

COROLlaRY 5.2. Let $f$ be parabolic, let $g$ be elliptic of order 2 , and let $\gamma=\gamma(f, g)$. If one of the following conditions holds, then $\langle f, g\rangle$ is discrete and isomorphic to the free product $\mathbb{Z} * \mathbb{Z}_{2}$.

\begin{tabular}{|c|c|c|c|c|c|}
\hline i) & $\gamma=\lambda i$ & $\lambda \geqslant 2$ & (ii) & $1 / 2+\lambda i$ & $\lambda \geqslant(2+\sqrt{3}) / 2$ \\
\hline & $\gamma=1+\lambda i$, & $\lambda \geqslant \sqrt{3}$ & (iv) & $3 / 2+\lambda i$, & $\lambda \geqslant \sqrt{7} / 2$ \\
\hline & $\gamma=2+\lambda i$ & $\lambda \geqslant 1$ & (vi) & $\gamma=5 / 2+\lambda i$, & $\lambda \geqslant \sqrt{3} / 2$ \\
\hline & $\begin{array}{l}\gamma=3+\lambda i, \\
\gamma=4+\lambda i\end{array}$ & $\begin{array}{l}\lambda \geqslant 1 / \sqrt{3} \\
\lambda \geqslant 0\end{array}$ & (viii) & $\begin{array}{l}\gamma=7 / 2+\lambda i, \\
\mathcal{R}(\gamma) \geq 4\end{array}$ & $\lambda \geqslant 1 / 2 \sqrt{3}$ \\
\hline
\end{tabular}

We now recall the following symmetries of the space of these 2-generator discrete groups. Namely, if $\gamma=\gamma(f, g)$ and $(\gamma, 0,-4)$ are the parameters of a discrete group, then so are $( \pm \gamma, 0,-4)$ and $( \pm \bar{\gamma}, 0,-4)$. All of these groups are isomorphic (in fact conjugate); this is a relatively straightforward observation, which we leave to the reader [9]. In particular, this observation enables us to restrict our attention to the positive quadrant, $\mathfrak{R}(\gamma) \geqslant 0$ and $\mathfrak{\Im}(\gamma) \geqslant 0$.

Now suppose that the parameter $\gamma$ gives rise to a nearly arithmetic Kleinian group generated by two parabolics. Then, by Theorem 3.4, $\gamma$ is a complex quadratic algebraic integer. Thus there are integers $b$ and $c$ such that $\gamma^{2}+b \gamma+c=0$, and we 
may assume $b^{2}-4 c<0$. Therefore $-7 \leqslant b \leqslant 0$, and Corollary 5.2 leaves us with the following possibilities for $\gamma$, listed in terms of increasing real part:

$$
\begin{gathered}
i, i \sqrt{2}, i \sqrt{3},(1+i \sqrt{3}) / 2,(1+i \sqrt{7}) / 2,(1+i \sqrt{11}) / 2, \\
1+i, 1+i \sqrt{2},(3+i \sqrt{3}) / 2 .
\end{gathered}
$$

Next, Riley's calculations concerning the moduli space of discrete groups generated by two parabolics [26] show that the points $(1+i \sqrt{11}) / 2$ and $1+i \sqrt{2}$ are well inside the 'free' part. His program 'Poincare' therefore shows that these groups are discrete and free on their generators. We wish to make two comments about the application of this computer program. First, discreteness is not an issue: we already know that each group in question is discrete [9]. Secondly, numerical or round-off error is not an issue, since we are presented in each case with a subgroup of $G_{d}=\operatorname{PSL}\left(2, O_{d}\right)$, and thus the numerical calculations can be done using exact arithmetic (though we have not done this).

There is an alternative approach, which we outline here. We do not give too many details, since we have adopted this approach in the paper [5] and more details are given there. The groups $G_{d}$ are finitely presented $[\mathbf{1 0}, \mathbf{2 8}]$, and it is not a difficult matter to find the matrices in question inside this group and to use a computational algebra package such as CAYLEY to verify that the subgroup in question is of infinite index (and therefore not arithmetic). For instance, for the value $1+\sqrt{2} i$, we find that the Bianchi group in question is $G_{2}$ with the presentation

$$
G_{2}=\left\langle a, b, c: b^{2}=(a b)^{3}=[a, c]=[b, c]^{2}=1\right\rangle .
$$

A matrix representation for this group in $\operatorname{PSL}(2, \mathbb{C})$ is given in $[\mathbf{1 0}]$. There we find that the group we are interested in is the subgroup $H=\langle a, b a c b\rangle$. We look for an intermediate subgroup $K$ with $H<K<G_{2}$ whose abelianisation has more than 2 independent infinite cyclic summands. If such a subgroup exists, then $H$ cannot be of finite index in $K$, and is therefore not of finite index in $G_{2}$. In the case outlined above, there is a subgroup of index 8 in $G_{2}$ which has this property. We thank M. D. E. Conder for help with CAYLEY in following this approach.

We are now left with 7 possible $\gamma$ values, and therefore there are at most 7 nearly arithmetic Kleinian groups generated by two parabolics, up to conjugacy. In fact, all these values do give rise to nearly arithmetic groups. We should also like to specifically identify the arithmetic groups. Thus let $G=\langle f, g\rangle$ be a Kleinian group with

$$
\operatorname{par}(G)=(\gamma, 0,-4),
$$

and set $G_{\gamma}=\langle f, g f g\rangle$ as the subgroup of index two generated by two parabolics with parameters

$$
\operatorname{par}\left(G_{\gamma}\right)=\left(\gamma^{2}, 0,0\right)
$$

The cases $\gamma=i, i \sqrt{2}, i \sqrt{3}$. Each of these three groups is nearly arithmetic, but not arithmetic. To see this, note that $\gamma(f, g f g)=-1,-2,-3$, respectively. Therefore $[f, g f g]$ is elliptic of order 3 in the first and last cases, and elliptic of order 2 in the second case. This shows that the groups are not free. When $\gamma(f, g f g)=-2,-3$, the group $\langle f, g f g\rangle$ is the generalised triangle group of type $(\infty, \infty ; 2)$ or $(\infty, \infty ; 3)$, respectively. These groups are discrete and faithful representations of the groups $\left\langle u, v: u^{\infty}=v^{\infty}=[u, v]^{p}=1\right\rangle$, where $p=2$ or 3 . Further information about these 
and related groups can be found in [11]. In particular, neither of these groups is arithmetic. The value $\gamma(f, g f g)=-1$ corresponds to a subgroup of infinite index in the index-two orientation-preserving subgroup of the group of reflections in a doubly truncated tetrahedron. These groups are discussed in $[\mathbf{8}]$ and have presentation

$$
\begin{aligned}
G_{\infty, \infty ; 3}^{+} \cong\langle u, v, w, s, t & : v^{2}=w^{2}=u^{3}=(u v)^{\infty}=(u w)^{\infty}=s^{2}=t^{2} \\
& \left.=(s u)^{2}=(u t)^{2}=(u w v)^{2}=(w t)^{2}=(s u v)^{2}=1\right\rangle,
\end{aligned}
$$

and $\langle f, g f g\rangle$ is conjugate to the subgroup generated by the parabolics $u v$ and $u w$. This subgroup is easily seen to be of infinite index, and is therefore not arithmetic.

The cases $\gamma=(1+i \sqrt{3}) / 2,(1+i \sqrt{7}) / 2,1+i,(3+i \sqrt{3}) / 2$. These four cases all correspond to arithmetic groups. The resulting cusped manifolds are two-bridge knot and link complements. It is conjectured that any finite-volume hyperbolic manifold whose fundamental group is generated by two parabolics is such a knot or link [16]. Recall that a non-elementary Kleinian group $G$ generated by two non-commuting parabolic elements can be normalised such that the generators have the form

$$
f=\left(\begin{array}{ll}
1 & 1 \\
0 & 1
\end{array}\right), \quad h=\left(\begin{array}{ll}
1 & 0 \\
z & 1
\end{array}\right) .
$$

Then $\gamma(f, h)=z^{2}$, and the extension $H$ of $G$ by an element $g$ of order 2 such that $H=\langle f, g\rangle$ has $\gamma(f, g)=z$. Using the standard notation $(p / q)$ for two-bridge knots or links, and the standard presentation for their groups [4], we find the value for $z$ in the following cases.
A. $\left(\frac{5}{3}\right) \quad z=\frac{1+i \sqrt{3}}{2}$
C. $\left(\frac{10}{3}\right) \quad z=\frac{3+i \sqrt{3}}{2}$
B. $\left(\frac{8}{3}\right) \quad z=1+i$
D. $\left(\frac{12}{5}\right) \quad z=\frac{1+i \sqrt{7}}{2}$

Note that from the normalisation given above, each of these groups will be a subgroup of the corresponding Bianchi group $G_{d}$.

A. This two-bridge knot is the figure- 8 knot, and the corresponding group is a subgroup of index 12 in $G_{3}$. It is known to be the only arithmetic knot [25].

B. This is the Whitehead link complement, which is also known to have index 12 in the Picard group $G_{1}$ (see, for example, [21]).

C. This is the link $6_{2}^{2}$ in the tables in [27]. It can be shown that the complement of this link can be obtained by suitably identifying faces of four ideal tetrahedra so that the uniformising group has index 24 in $G_{3}$.

D. This is the link $6_{3}^{2}$ in the tables in [27]. Also, the once-punctured torus bundle with monodromy $L^{2} R$ with generators $a, b, t$ as described in [3] has a subgroup of index 2 generated by parabolics $t, a t a^{-1}$. Now $\gamma(t, a)^{2}=\gamma\left(t, a t a^{-1}\right)=\frac{1+i \sqrt{7}}{2}$. Thus the link complement is a 2 -sheeted cover of the once-punctured torus bundle with monodromy $L^{2} R$.

\section{References}

1. A. F. BEARDON, The geometry of discrete groups (Springer, New York, 1983).

2. A. Borel, 'Commensurability classes and volumes of hyperbolic 3-manifolds', Ann. Scuola Norm. Sup. Pisa 8 (1981) 1-33.

3. B. Bowditch, C. Maclachlan and A. W. Reid, 'Arithmetic surface bundles', Math. Ann. 302 (1995) 31-60.

4. G. Burde and H. Zieschang, Knots (de Gruyter, Berlin, 1985). 
5. M. D. E. Conder, C. Maclachlan, G. J. Martin and E. O'Brien, '2-generator arithmetic Kleinian groups, III', Preprint.

6. F. W. Gehring, C. Maclachlan and G. J. Martin, 'On discrete free products of cyclic groups' Math. Sem. Geissen 228 (1996) 9-15.

7. F. W. Gehring, C. Maclachlan, G. J. Martin and A. W. Reid, 'Arithmeticity, discreteness and volume', Trans. Amer. Math. Soc. 349 (1997) 3611-3643.

8. F. W. Gehring, T. H. Marshall and G. J. Martin, 'On the spectrum of axial distances in Kleinian groups', Preprint.

9. F. W. Gehring and G. J. Martin, 'Commutators, collars and the geometry of Möbius groups', $J$. Anal. Math. 63 (1994) 175-219.

10. F. Grunewald and J. SChwermer, 'Subgroups of Bianchi groups and arithmetic quotients of hyperbolic 3-space', Trans. Amer. Math. Soc. 335 (1993) 47-78.

11. M. Hagelberger, C. Maclachlan and G. Rosenberger, 'On discrete generalised triangle groups', Proc. Edinburgh Math. Soc. 38 (1995) 397-412.

12. H. M. Hilden, M-T. Lozano and J. M. Montesinos, 'A characterization of arithmetic subgroups of SL $(2, \mathbf{R})$ and SL(2,C)', Math. Nachr. 159 (1992) 245-270.

13. J. I. Ignatov, 'Free and nonfree subgroups of $\operatorname{PSL}(2, \mathbb{C})$ generated by two parabolic elements', Math. USSR Sb. 35 (1979) 49-55.

14. L. Keen and C. Series, 'Pleating co-ordinates for the Maskit embedding of the Teichmüller space of punctured tori', Topology 32 (1993) 719-749.

15. L. KeEn and C. Series, 'The Riley slice of Schottky space', Proc. London Math. Soc. 69 (1994) 72-90.

16. R. Kirby, 'Problems in low-dimensional topology', Geometric topology Part 2: Studies in advanced maths (ed. W. Kazez, AMS/IP, 1996).

17. R. C. Lyndon and J. L. Ullman, 'Groups generated by two parabolic linear fractional transformations', Canad. J. Math. 21 (1969) 1388-1403.

18. C. Maclachlan and G. J. Martin, '2-generator arithmetic Kleinian groups', Preprint.

19. C. Maclachlan and A. W. ReID, 'Commensurability classes of arithmetic Kleinian groups and their Fuchsian subgroups', Math. Proc. Cambridge Philos. Soc. 102 (1987) 251-257.

20. B. MASKIT, Kleinian groups (Springer, Berlin, 1987).

21. W. D. Neumann and A. W. ReID, 'Arithmetic of hyperbolic 3-manifolds', Topology '90, Proceedings of the Research Semester in Low-dimensional Topology at Ohio State University (ed. B. Apanasov, W. D. Neumann, A. W. Reid and L. Siebenmann, de Gruyter, Berlin, 1992) 273-310.

22. J. R. PARKER, 'Kleinian groups with two parabolic generators', Preprint.

23. J. G. RATCLIFFE, Foundations of hyperbolic manifolds (Springer, New York, 1994).

24. A. W. ReID, 'A note on trace-fields of Kleinian groups', Bull. London Math. Soc. 22 (1990) 349-352.

25. A. W. ReID, 'Arithmeticity of knot complements', J. London Math. Soc. (2) 43 (1991) 171-184.

26. R. Riley, 'Applications of a computer implemenetation of Poincaré's theorem on fundamental polyhedra', Math. Comp. 40 (1983) 607-632.

27. D. Rolfsen, Knots and links (Publish or Perish, Berkeley, 1976).

28. R. Swan, 'Generators and relations for certain special linear groups', Adv. Math. 6 (1971) 1-78.

29. K. TAKEUCHI, 'Arithmetic triangle groups', J. Math. Soc. Japan 29 (1977) 91-106.

30. W. P. Thurston, The geometry and topology of three-manifolds, Princeton Univ. Lecture Notes (Princeton University Press, Princeton, NJ, 1976).

31. M-F. Vignéras, Arithmétique des algèbres de quaternions, Lecture Notes in Math. 800 (Springer, Berlin, 1980).

F. W. Gehring

University of Michigan

Ann Arbor, MI 48109

USA

\section{G. J. Martin}

University of Auckland

Auckland

New Zealand
C. Maclachlan

University of Aberdeen

Aberdeen AB24 3UE

Scotland 